Sophia Haussener

Department of Mechanical and Process

Engineering,

ETH Zurich,

8092 Zurich, Switzerland

Patrick Coray

Solar Technology Laboratory,

Paul Scherrer Institute,

5232 Villigen, Switzerland

Wojciech Lipiński

Department of Mechanical Engineering,

University of Minnesota,

Minneapolis, MN 55455

Peter Wyss

Department of Electronics/Metrology,

EMPA Material Science and Technology,

Überlandstrasse 129,

8600 Dübendorf, Switzerland

Aldo Steinfeld ${ }^{1}$

Department of Mechanical and Process

Engineering,

ETH Zurich,

8092 Zurich, Switzerland;

and

Solar Technology Laboratory,

Paul Scherrer Institute,

5232 Villigen, Switzerland

e-mail: aldo.steinfeld@ethz.ch

\section{Tomography-Based Heat and Mass Transfer Characterization of Reticulate Porous Ceramics for High-Temperature Processing}

Reticulate porous ceramics employed in high-temperature processes are characterized for heat and mass transfer. The exact 3D digital geometry of their complex porous structure is obtained by computer tomography and used in direct pore-level simulations to numerically calculate their effective transport properties. Two-point correlation functions and mathematical morphology operations are applied for the geometrical characterization that includes the determination of porosity, specific surface area, representative elementary volume edge size, and mean pore size. Finite volume techniques are applied for conductive/convective heat transfer and flow characterization, which includes the determination of the thermal conductivity, interfacial heat transfer coefficient, permeability, Dupuit-Forchheimer coefficient, residence time, tortuosity, and diffusion tensor. Collision-based Monte Carlo method is applied for the radiative heat transfer characterization, which includes the determination of the extinction coefficient and scattering phase function. [DOI: 10.1115/1.4000226]

Keywords: reticulate, porous, ceramic, computer tomography, transport, radiation, conduction, convection, Monte Carlo, DPLS, solar energy, thermochemical cycle, hydrogen

\section{Introduction}

Reticulate porous ceramics (RPCs) exhibit favorable heat and mass transfer characteristics for high-temperature applications [1]. Examples are molten-metal and diesel-engine-exhaust filters [2,3], radiant burners [4], catalyst supports [5], and radiant absorbers in solar thermal and thermochemical reactors [6]. In the latter application, RPCs provide efficient absorption of concentrated solar radiation and large specific surface areas for heterogeneous chemical reactions [6-8]. The accurate knowledge of the RPCs' effective heat and mass transport properties is crucial for their optimal design and operation. Computer tomography $(\mathrm{CT})$ enables the digital geometrical representation of their complex porous structure, which is needed for the direct pore-level numerical simulations (DPLSs).

Previous pertinent studies include the determination of the extinction coefficient, scattering coefficient, and scattering phase function of metal foams and RPCs for simplified geometries composed of pentagon dodecahedron or tetracaedecaedric [9] and spherical voided cubic unit cells [10]. The effective thermal conductivity was determined for simplified foam geometries composed of tetrakaidecahedron [11], hexagonal [12], and spherical or cubical voided cubic unit cells [13]. Permeability has been determined by simplifying the foam structure by parallel conduits $[14]$ or by empirical correlations for fibrous beds $[15,16]$. The exact geometry of a porous magnetic gel sample, obtained by microCT,

\footnotetext{
${ }^{1}$ Corresponding author.

Contributed by the Heat Transfer Division of ASME for publication in the JourNAL OF Heat TRANSFER. Manuscript received October 31, 2008; final manuscript received April 29, 2009; published online December 3, 2009. Assoc. Editor: Yogesh Jaluria.
}

was used to compute the mean survival time, which was linked to the permeability by an empirical correlation [17]. More recently, CT-based methodology was applied to determine the effective transport properties of a Rh-coated SiC RPC used for the solar steam-reforming of $\mathrm{CH}_{4}$ [18-21] and for a packed bed of semitransparent, large $\mathrm{CaCO}_{3}$ particles [22].

In the present study, CT-based DPLS is applied to determine porosity, specific surface area, representative elementary volume, pore size distribution, extinction coefficient, scattering phase function, effective conductivity, heat transfer coefficient, permeability, Dupuit-Forchheimer coefficient, tortuosity, residence time, and dispersion tensor for an uncoated, nonhollow SiSiC RPC. This material was recently used in a solar reactor for the thermal decomposition of $\mathrm{H}_{2} \mathrm{SO}_{4}$ as part of a water-splitting thermochemical cycle [23]

\section{Morphological Characterization}

2.1 Low-resolution CT. The RPC foam sample, made of Si$\mathrm{SiC}$, is shown in Fig. 1. Its nominal pore diameter is $d_{\text {nom }}$ $=1.27 \mathrm{~mm}$, corresponding to 20 pores per in. (ppi). The sample is exposed to an unfiltered polychromatic X-ray beam generated by electrons incident on a wolfram target. The generator is operated at an acceleration voltage of $60 \mathrm{keV}$ and a current of $0.11 \mathrm{~mA}$. A Hamamatsu flatpanel C7942 CA-02 protected by a $0.1 \mathrm{~mm}$-thick aluminum filter is used to detect the transmitted X-rays. The sample is scanned at 900 angles (projections). Each projection consists of an average over six scans, each with an exposure time of $0.7 \mathrm{~s}$. Low-resolution computer tomography (LRCT) is performed for voxel sizes of $30 \mu \mathrm{m}$ and $15 \mu \mathrm{m}$ and field of view of $2.3 \times 2.3 \times 1.2 \mathrm{~cm}^{3}$ and $1.2 \times 1.2 \times 0.6 \mathrm{~cm}^{3}$, respectively.

$3 \mathrm{D}$ surface rendering of a sample subvolume, reconstructed 


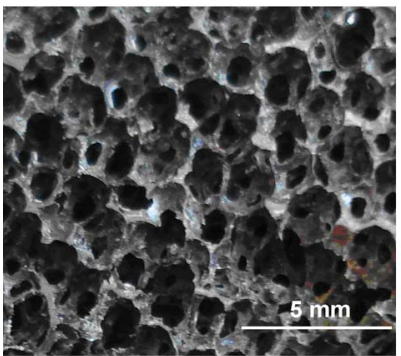

(a)

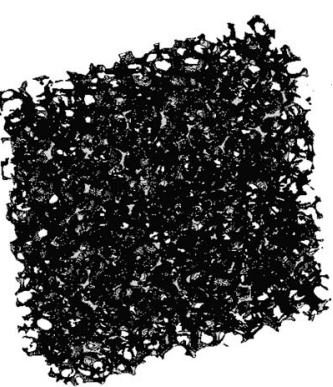

(b)
Fig. 1 Sample of RPC foam: (a) top view photograph and (b) 3D surface rendering of $15 \mu \mathrm{m}$ voxel size tomography data

from the resulting tomography data, is depicted in Fig. 1(b). The histograms of the normalized absorption values, as shown in Fig. 2 , have a bimodal character for both scan resolutions. The mode method is applied for phase segmentation [24,25]. The normalized phase threshold values $\alpha_{\mathrm{t}} / \alpha_{\max }$ are 0.39 and 0.23 for scans with voxel sizes of $30 \mu \mathrm{m}$ and $15 \mu \mathrm{m}$, respectively. These results are comparable to $\alpha_{\mathrm{t}} / \alpha_{\max }=0.35$ and 0.20 for scans with voxel sizes of $30 \mu \mathrm{m}$ and $15 \mu \mathrm{m}$, respectively, calculated using Otsu's method [24]. $\alpha_{\mathrm{t}} / \alpha_{\max }$ varies by \pm 0.04 , as corroborated for three selected tomograms divided into 36 subelements and for voxel sizes of $15 \mu \mathrm{m}$ and $30 \mu \mathrm{m}$, shown in Fig. 3 .

2.2 High-resolution CT. Figure 4(a) shows a tomogram of a single RPC's strut obtained by high-resolution computer tomography (HRCT) of $0.37 \mu \mathrm{m}$ voxel size and $0.76 \times 0.76$ $\times 0.62 \mathrm{~mm}^{3}$ field of view. The HRCT is performed on the TOMCAT beamline at the Swiss Light Source (SLS) of the Paul Scherrer Institute (Villigen, Switzerland) [26] for $14 \mathrm{keV}$ photon energy, $400 \mu \mathrm{A}$ beam current, $100 \mu \mathrm{m}$ thick aluminum filter, $20 \times$ geometrical magnification, $0.8 \mathrm{~s}$ exposure time, and 1501 projections. A magnified fragment of the strut edge is shown in Fig. 4(b). The strut surface is irregularly shaped. Irregular spatial distribution of $\mathrm{SiC}$ and $\mathrm{Si}$ within the strut leads to internal heterogeneity but no pores are observed inside the strut.

2.3 Porosity and Specific Surface. The two-point correlation function

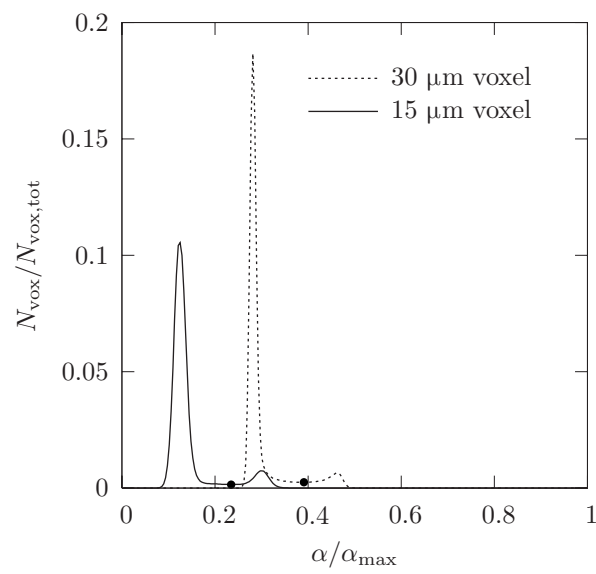

Fig. 2 Normalized histograms of the absorption values for the scans with $30 \mu \mathrm{m}$ (dotted line) and $15 \mu \mathrm{m}$ (solid line) voxe sizes. The points indicate the corresponding threshold values of $\alpha_{\mathrm{t}} / \alpha_{\max }=0.39$ and 0.23 .

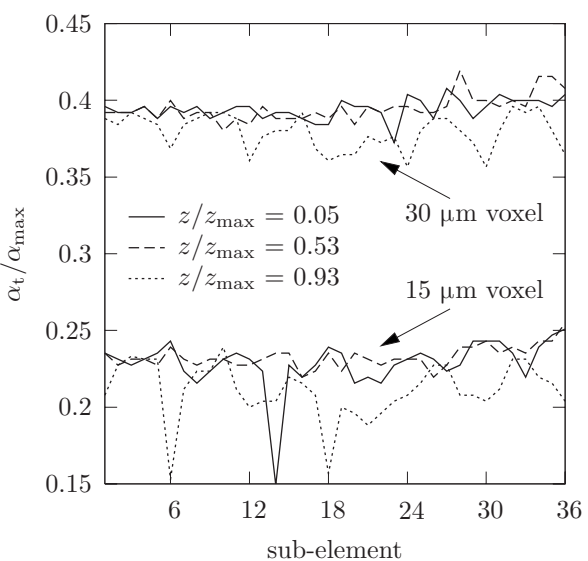

Fig. 3 Normalized threshold absorption values for 36 subelements of three selected tomograms for voxel sizes of $30 \mu \mathrm{m}$ and $15 \mu \mathrm{m}$

$$
s_{2}(r)=\frac{\int_{V} \int_{4 \pi} \psi(\mathbf{r}) \psi(\mathbf{r}+r \hat{\mathbf{s}}) \mathrm{d} \Omega \mathrm{d} V}{4 \pi V}
$$

with its properties $s_{2}(0)=\varepsilon$ and $\left.(\mathrm{d} s / \mathrm{d} r)\right|_{r=0}=-\left(A_{0} / 4\right)$ [27] is applied. $s_{2}(r)$ is computed using the Monte Carlo method. A random point is chosen within the fluid phase. A second random point is chosen at distance $r$. If the second point belongs to the fluid phase, the integrand in Eq. (1) is equal to 1. Otherwise, it is 0 . The computation is performed for $10^{8}$ random points and for $r$ varying between $0 \mathrm{~cm}$ and $1 \mathrm{~cm}$. The resulting porosity is $\varepsilon=0.91$. It compares well to the value obtained by weight measurement $(0.90 \pm 0.02)$ and it does not depend on the voxel size of the scan. In contrast, the specific surface area $A_{0}$ increases from $1367 \mathrm{~m}^{-1}$ to $1680 \mathrm{~m}^{-1}$ as the voxel size decreases from $30 \mu \mathrm{m}$ to $15 \mu \mathrm{m}$ because of the better resolution of surface irregularities for the smaller voxel size.

2.4 REV. The representative elementary volume (REV) is defined as the minimum volume of a porous material for which the continuum assumption is valid. It is determined based on porosity calculations for subsequent growing volumes until it asymptotically reaches a constant value within a band of $\pm \gamma$. The edge length of the cubic REV is defined by [18]

$$
l_{\mathrm{REV}, \gamma}=\min \left\{l \leq l^{*} \mid \varepsilon-\gamma<\varepsilon\left(S_{l^{*}}\right)<\varepsilon+\gamma\right\}, \quad \gamma<1
$$

where $S_{l^{*}}$ is a sample subvolume of edge length $l^{*}$. For $\gamma=0.02$, $l_{\mathrm{REV}, \gamma}=3.50 \mathrm{~mm}$ and $3.39 \mathrm{~mm}$ for the scans with voxel sizes $30 \mu \mathrm{m}$ and $15 \mu \mathrm{m}$, respectively, corresponding to $2.76 d_{\text {nom }}$ and $2.67 d_{\text {nom }}$, respectively. It is shown in the analysis that follows

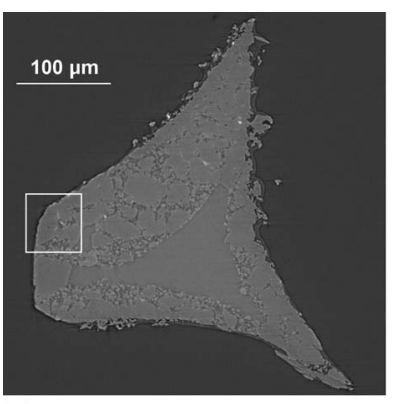

(a)

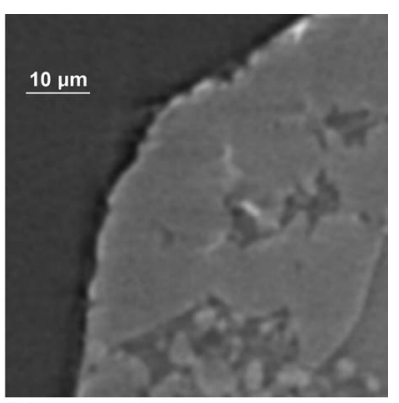

(b)
Fig. 4 (a) Tomogram of a single strut obtained by HRCT; $(b)$ magnified fragment of the strut edge marked with the white frame in Fig. 4(a) 


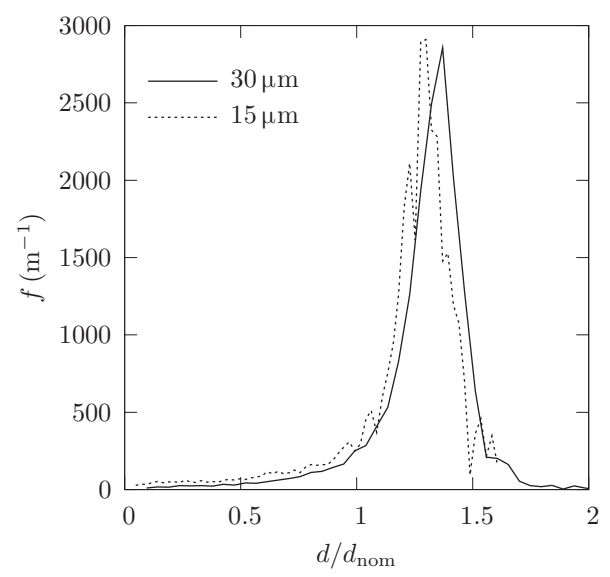

Fig. 5 Opening pore size distribution of the RPC foam for the $30 \mu \mathrm{m}$ (solid curve) and $15 \mu \mathrm{m}$ (dotted curve) voxel size tomography data

(Sec. 3.4) that at least $l_{\mathrm{REV}}=3.50 d_{\mathrm{nom}}$ is required for computations of heat and mass transfer.

2.5 Pore Size Distribution. An opening operation, consisting of erosion, followed by dilation with structuring sphere of diameter $d$, is applied to compute the opening porosity $\varepsilon_{\mathrm{op}}$ as a function of the diameter $d$. $\varepsilon_{\text {op }}$ is then used to determine the RPC's pore size distribution function $f[18]$

$$
F(d)=\int_{0}^{d} f\left(d^{*}\right) \mathrm{d} d^{*}=1-\frac{\varepsilon_{\text {op }}(d)}{\varepsilon_{0}}
$$

where $\varepsilon_{0}=s_{2}(0)$. The resulting pore size distribution functions are shown in Fig. 5 for the $30 \mu \mathrm{m}$ and $15 \mu \mathrm{m}$ voxel size tomography data. Table 1 lists the median, mode, mean, and hydraulic $\left(d_{\mathrm{h}}\right.$ $\left.=4 \varepsilon / A_{0}\right)$ diameters resulting from the distributions in Fig. 5 .

Good qualitative and quantitative agreement of porosity, specific surface area, and pore size distribution is obtained for voxel sizes of $30 \mu \mathrm{m}$ and $15 \mu \mathrm{m}$. Thus, the heat and mass transfer characteristics in Secs. 3 and 4 are computed by using the $30 \mu \mathrm{m}$ resolution tomography data.
Table 1 Arithmetic mean, mode, median, and hydraulic diameter for the $30 \mu \mathrm{m}$ and $15 \mu \mathrm{m}$ voxel size tomography data

\begin{tabular}{ccccc}
\hline \hline $\begin{array}{c}\text { Voxel size } \\
(\mu \mathrm{m})\end{array}$ & $\begin{array}{c}d_{\mathrm{am}} \\
(\mathrm{mm})\end{array}$ & $\begin{array}{c}d_{\text {mode }} \\
(\mathrm{mm})\end{array}$ & $\begin{array}{c}d_{\text {median }} \\
(\mathrm{mm})\end{array}$ & $\begin{array}{c}d_{\mathrm{h}} \\
(\mathrm{mm})\end{array}$ \\
\hline 30 & 1.64 & 1.74 & 1.69 & 2.76 \\
15 & 1.55 & 1.65 & 1.62 & 2.24 \\
\hline \hline
\end{tabular}

\section{Heat Transfer Characteristics}

3.1 Radiative Properties. The complex refractive index of $\mathrm{SiC}$ is taken for the solid phase [28], which is assumed to be opaque in the visible and near infrared spectral region. The fluid phase is assumed to be radiatively nonparticipating. Hence, the governing equation of radiative transfer is $[19,22,29,30]$

$$
\frac{\mathrm{d} I_{\lambda}(s, \hat{\mathbf{s}})}{\mathrm{d} s}+\beta_{\lambda} I_{\lambda}(s, \hat{\mathbf{s}})=\kappa_{\lambda} I_{\lambda, \mathrm{b}}(s, \hat{\mathbf{s}})+\frac{\sigma_{\mathrm{s}, \lambda}}{4 \pi} \int_{4 \pi} I_{\lambda}\left(s, \hat{\mathbf{s}}_{\mathrm{in}}\right) \Phi_{\lambda}\left(\hat{\mathbf{s}}, \hat{\mathbf{s}}_{\mathrm{in}}\right) \mathrm{d} \Omega_{\mathrm{in}}
$$

where $I_{\lambda}$ is spectral radiative intensity, $\kappa_{\lambda}, \sigma_{\mathrm{s}, \lambda}$, and $\beta_{\lambda}$ are the spectral absorption, scattering, and extinction coefficients, respectively, and $\Phi_{\lambda}$ is the spectral scattering phase function. The spectral subscript $\lambda$ is omitted in the following text for brevity. The characteristic size parameter $\left(\pi d_{\mathrm{h}} / \lambda\right)$ is much larger than 1 ; thus, geometric optics is valid. Collision-based Monte Carlo method is applied to compute the cumulative distribution functions of the radiation attenuation path $s$, and the distribution function of the cosine of incidence $\mu_{\text {in }}$ at the solid wall $G_{\mathrm{e}}(s)$ and $F_{\mu_{\text {in }}}\left(\mu_{\text {in }}\right)$, respectively

$$
\begin{gathered}
G_{\mathrm{e}}(s)=\int_{0}^{s} \frac{1}{N_{\mathrm{r}}} \sum_{j=1}^{N_{\mathrm{r}}} \delta\left(s^{\prime}-s_{j}^{\prime}\right) \mathrm{d} s^{\prime} \\
F_{\mu_{\mathrm{in}}}\left(\mu_{\mathrm{in}}\right)=\frac{1}{N_{\mathrm{e}}} \sum_{j=1}^{N_{\mathrm{e}}} \delta\left(\mu_{\mathrm{in}}-\mu_{\mathrm{in}, j}\right)
\end{gathered}
$$

$G_{\mathrm{e}}(s)$ and $F_{\mu_{\text {in }}}\left(\mu_{\text {in }}\right)$ are related to $\beta$ and $\Phi$ by $[19,22,29]$

$$
G_{\mathrm{e}}(s) \approx 1-\exp (-\beta s)
$$

where $\mu_{\mathrm{s}}$ and $\mu_{\mathrm{r}}$ denote the cosines of scattering and reflection angles. $G_{\mathrm{e}}(s)$ and $F_{\mu_{\text {in }}}\left(\mu_{\text {in }}\right)$ are computed by following the histories of a large number $\left(N_{\mathrm{r}}=6 \times 10^{6}\right)$ of stochastic rays launched at random locations within the fluid phase of the REV. $N_{\mathrm{e}}$ rays interact with the solid-fluid interface by either absorption or reflection. The path length $s$ within REV is recorded for all $N_{\mathrm{r}}$ rays while the cosine of incidence $\mu_{\text {in }}$ is recorded for all $N_{\mathrm{e}}$ rays $[19,22,29,30]$. A sample of $18 \times 18 \times 12 \mathrm{~mm}^{3}$, corresponding to $600 \times 600 \times 400$ voxels, is investigated. The intersection point between a ray and the solid-fluid interface is found by following the ray in discrete steps until the normalized gray values are larger than $\alpha_{\mathrm{t}} / \alpha_{\max }$. Finally, the exact determination is achieved by applying the bisection method. Note that the interface is described by a continuous function and no numerical grid is required. The computations are performed with an in-house Fortran 95 code.
$1-G_{\mathrm{e}}(s)$ is plotted in Fig. 6(a) as a function of the normalized path length for two values of the threshold $\alpha_{\mathrm{t}} / \alpha_{\max }: 0.31$ and 0.39 . A least-square fit to Bouguer's law (Eq. (7)), also shown in Fig. 6(a), yields a constant extinction coefficient $\beta_{\mathrm{MC}}=628.4 \mathrm{~m}^{-1}$ with root mean square (RMS) of $0.014 \mathrm{~m}^{-1}$ for $\alpha_{\mathrm{t}} / \alpha_{\max }=0.31$, and $\beta_{\mathrm{MC}}=430.8 \mathrm{~m}^{-1}$ with RMS $=0.014 \mathrm{~m}^{-1}$ for $\alpha_{\mathrm{t}} / \alpha_{\max }=0.39$. Separate computations along preferred directions showed slight anisotropy of $\beta$, as indicated in Table 2 .

For assumed diffusely-reflecting surface of the solid phase, the scattering phase function is plotted in Fig. $6(b)$ as a function of the cosine of scattering angle. It is well approximated by (RMS $=0.010)$

$$
\Phi=0.5471 \mu_{\mathrm{s}}^{2}-1.38838 \mu_{\mathrm{s}}+0.8176
$$

Also shown in Fig. $6(b)$ is the analytically determined scattering phase function for diffusely-reflecting large opaque spheres [31] 

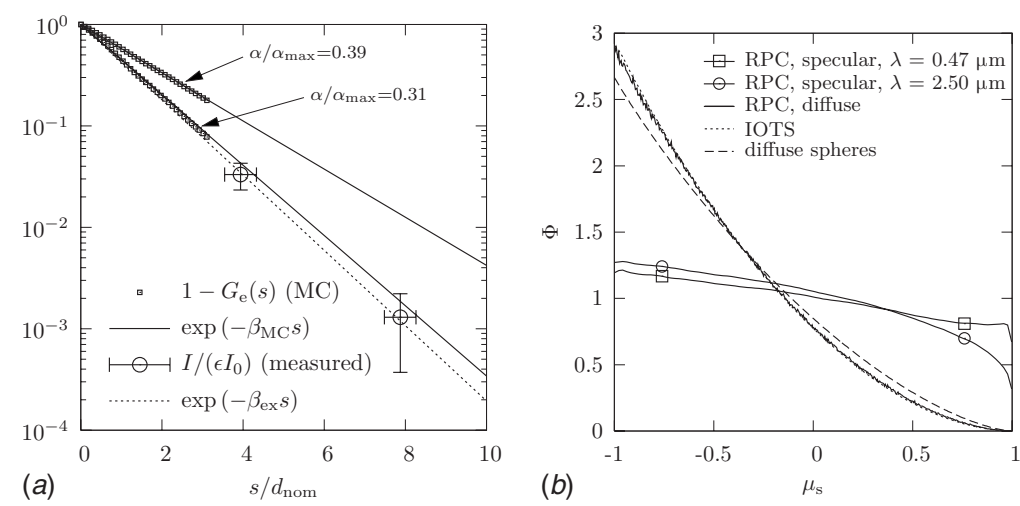

Fig. 6 (a) Variation in computed and measured incident radiative intensities as a function of normalized path length in the sample; (b) scattering phase functions of the RPC foam, IOTS, and of large diffuse opaque spheres as a function of the cosine of scattering angle

The RPC foam and identical overlapping transparent spheres (IOTS) [30] exhibit identical scattering behavior due to their morphological similarity. Compared with large diffuse opaque spheres, RPC exhibits enhanced scattering in backward direction and less in forward direction. For assumed specularly-reflecting surface, two exemplary scattering phase functions computed for $m_{\lambda=0.467 \mu \mathrm{m}}=2.707+1.46 i$ and $m_{\lambda=2.50 \mu \mathrm{m}}=2.562+5.17 i$ are shown in Fig. $6(b)$. Both exhibit nearly isotropic scattering behaviors with slightly increased backward scattering. Based on the irregular surface topography shown in Fig. 4, the solid phase is anticipated to show a dominant diffuse component in the reflection pattern.

The scattering albedo $\sigma_{\mathrm{s}} / \beta$ equals the surface reflectivity of the solid phase, assumed to be wavelength-independent and equal to 0.1 [32]. Hence, $\sigma_{\mathrm{s}}=43.1 \mathrm{~m}^{-1}$ and $62.8 \mathrm{~m}^{-1}$ for $\alpha_{\mathrm{t}} / \alpha_{\max }=0.31$ and 0.39 , respectively.

The extinction coefficient is independently estimated based on experimental measurements by using the spectroscopy system shown in Fig. 7 [33]. The main hardware components of the setup are as follows: (1) a dual Xe-Arc/Cesiwid-Glowbar lamp as a source of radiation, (2) a double monochromator (Acton Research Spectra Pro Monochromator SP-2355 series) with monochromator exit slit $\left(2^{\prime}\right),\left((3)\right.$ and (5)) two imaging lens pairs $\left(\mathrm{MgF}_{2}\right.$, focal lengths $f=75 \mathrm{~mm}$ and $f=150 \mathrm{~mm}$ ), (4) a sample holder, (6) a detector (Si/PC-HgCdTe sandwich with thermoelectric cooler), (7) an optical chopper to modulate the radiation leaving the monochromator, (8) a lock-in amplifier to measure the modulated signal, and (9) a PC data acquisition system. This setup enables measurements in the spectral range from $0.3 \mu \mathrm{m}$ to $4 \mu \mathrm{m}$ with a spectral resolution of $\pm 1 \mathrm{~nm}$ and an angular resolution of $10 \mathrm{deg}$. The maximum acceptance angle for detection of an incoming ray measured with respect to the optical axis is less than $4 \mathrm{deg}$. Angular measurements are performed with two RPC foam samples of thicknesses $5 \mathrm{~mm}$ and $10 \mathrm{~mm}$ at radiation wavelengths of $0.3 \mu \mathrm{m}, 0.6 \mu \mathrm{m}$, and $0.9 \mu \mathrm{m}$. The measured flux rapidly decreases with the increasing detection angle. At $10 \mathrm{deg}$, it is $10^{3}$ smaller than that acquired in the forward direction. Since the reflectivity of the solid phase is comparable to that of $\mathrm{SiC}(0.1)$ [32] the contribution of the incoming scattering to the measured radia-

Table 2 Mean values and standard deviations of the extinction coefficient along three directions

\begin{tabular}{lrrc}
\hline \hline & $x$-direction & $y$-direction & $z$-direction \\
\hline$\beta_{\mathrm{MC}}$, mean $\left(\mathrm{m}^{-1}\right)$ & 400.6 & 411.0 & 439.8 \\
$S_{\beta}\left(\mathrm{m}^{-1}\right)$ & 35.8 & 37.5 & 28.4 \\
\hline \hline
\end{tabular}

tive fluxes is neglected. Thus, the extinction coefficient is estimated by assuming Bouguer's law-type dependency of the measured radiative fluxes on the sample thickness.

For all radiation wavelengths, the extinction coefficient is approximately constant and equal to $\beta_{\mathrm{ex}}=673 \pm 30 \mathrm{~m}^{-1}$. This value is larger than $\beta_{\mathrm{MC}}=430.8 \mathrm{~m}^{-1}$ determined numerically by $\mathrm{MC}$ for $\alpha_{\mathrm{t}} / \alpha_{\max }=0.39$, but it is in good agreement with $\beta_{\mathrm{MC}}$ $=628.4 \mathrm{~m}^{-1}$ computed for $\alpha_{\mathrm{t}} / \alpha_{\max }=0.31$. Since $1 / \sigma_{\mathrm{s}}$ has the same order of magnitude as the measured sample thickness in coming scattering may affect the experimental results. In contrast, when comparing measured and calculated porosities, $\left(\varepsilon_{\mathrm{ex}}\right.$ $\left.-\varepsilon_{\mathrm{CT}}\right) / \varepsilon_{\mathrm{ex}}=0.01$ and 0.06 for $\alpha_{\mathrm{t}} / \alpha_{\max }=0.39$ and 0.31 , respectively.

3.2 Effective Thermal Conductivity. The governing steadystate heat conduction equations within the solid and fluid phases are

$$
\begin{aligned}
& 0=\nabla\left(k_{\mathrm{s}} \nabla T_{\mathrm{s}}\right) \\
& 0=\nabla\left(k_{\mathrm{f}} \nabla T_{\mathrm{f}}\right)
\end{aligned}
$$

The boundary conditions are

$$
T_{\mathrm{s}}=T_{\mathrm{f}}, \hat{\mathbf{n}} \cdot k_{\mathrm{s}} \nabla T_{\mathrm{s}}=\hat{\mathbf{n}} \cdot k_{\mathrm{f}} \nabla T_{\mathrm{f}} \text { at solid-fluid interface }
$$

$\mathbf{q}^{\prime \prime} \cdot \hat{\mathbf{n}}=0$ at the sample lateral walls

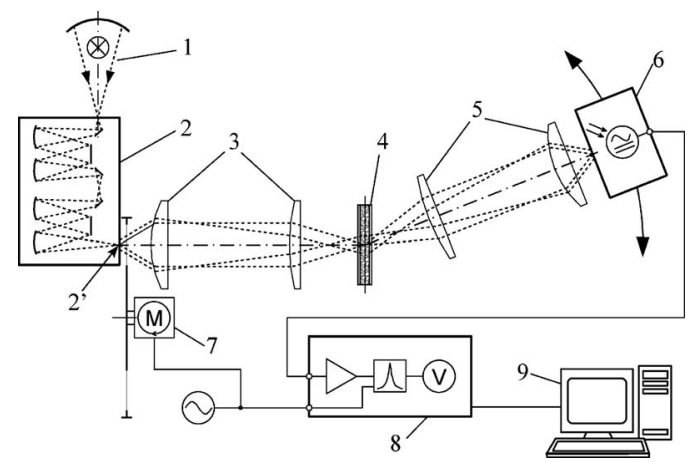

Fig. 7 Experimental spectroscopy setup: (1) dual Xe-Arc/ Cesiwid-Glowbar lamp, (2) double monochromator, ((3) and (5)) collimating and focusing lens pairs, (4) sample mounted on a linear positioning stage, (6) detector, (7) optical chopper, (8) lock-in amplifier, (9) data acquisition system 

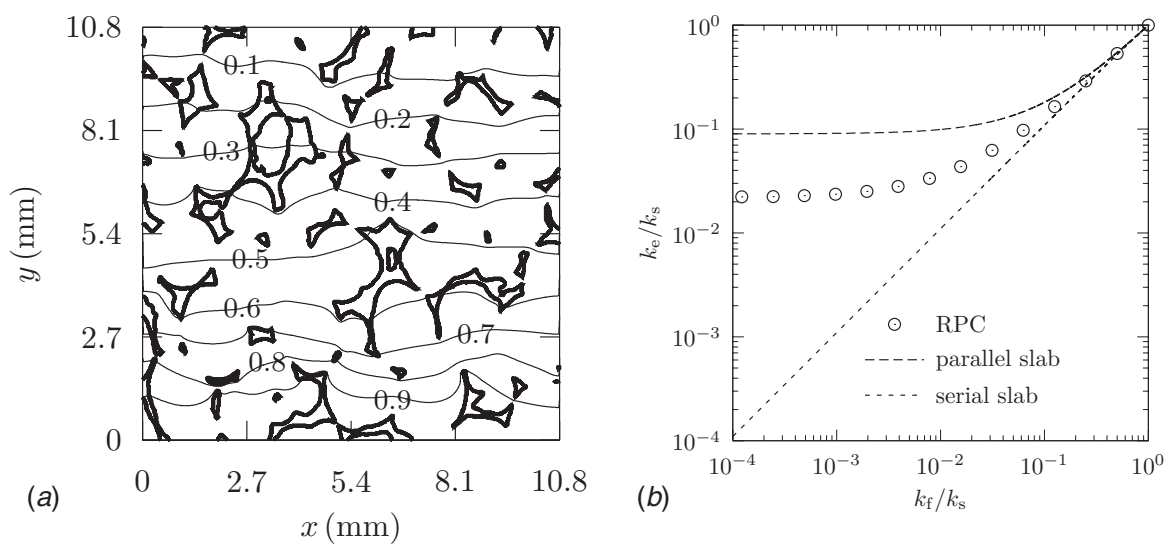

Fig. 8 (a) Contour map of the normalized temperature distribution ( $T$ $\left.-T_{2}\right) /\left(T_{1}-T_{2}\right)$ along the axis perpendicular to the temperature boundary condition of the RPC foam (thick solid lines depict solid-fluid phase boundary) for $k_{\mathrm{f}} / k_{\mathrm{s}}=1.0 \times 10^{-4}$; (b) the effective thermal conductivity of the RPC foam and of parallel and serial slabs at $\varepsilon=0.91$

$$
\begin{gathered}
T_{\mathrm{s}}=T_{\mathrm{f}}=T_{1} \quad \text { at the sample inlet } \\
T_{\mathrm{s}}=T_{\mathrm{f}}=T_{2} \text { at the sample outlet }
\end{gathered}
$$

The finite volume (FV) technique with successive over-relaxation (SOR) is applied to solve the Eqs. (10) and (11). The computations are performed with an in-house Fortran 95 code. A sample of $10.8 \times 10.8 \times 10.8 \mathrm{~mm}^{3}$, corresponding to $360 \times 360 \times 360$ voxels, is investigated. Grid convergence is obtained with mesh element size of $21.5 \mu \mathrm{m}$. The boundary conditions Eqs. (12)-(15) lead to a quasi-1D temperature profile. A contour map of the normalized temperature distribution along the axis perpendicular to the temperature boundary condition is shown in Fig. 8(a). The nonlinear temperature profile in the sample is used to determine the heat flux across the sample at any given cross-sectional plane perpendicular to the main heat flow direction. By applying the one-equation average model describing the conduction heat transfer in porous media $[14,20]$, the heat flux can be linked to the effective conductivity by

$$
k_{\mathrm{e}}=l \frac{-\int_{A_{\mathrm{s}}} k_{\mathrm{s}} \nabla T_{\mathrm{s}} \cdot \hat{\mathbf{n}} \mathrm{d} A_{\mathrm{s}}-\int_{A_{\mathrm{f}}} k_{\mathrm{f}} \nabla T_{\mathrm{f}} \cdot \hat{\mathbf{n}} \mathrm{d} A_{\mathrm{f}}}{\left(T_{1}-T_{2}\right)\left(A_{\mathrm{s}}+A_{\mathrm{f}}\right)}
$$

The effective sample conductivity as a function of the solid and fluid conductivity is shown in Fig. 8(b). It is compared with the parallel and serial slab assumptions (at $\varepsilon=0.91$ ), which indicate minimal and maximal possible conductivities.

The computed $k_{\mathrm{e}} / k_{\mathrm{s}}$ was fitted to the linear combination of thermal conductivities of parallel and serial slabs [14]

$$
\frac{k_{\mathrm{e}}}{k_{\mathrm{s}}}=e_{1} \frac{k_{\mathrm{f}} / k_{\mathrm{s}}}{\varepsilon\left(1-k_{\mathrm{f}} / k_{\mathrm{s}}\right)+k_{\mathrm{f}} / k_{\mathrm{s}}}+e_{2}\left(\varepsilon \frac{k_{\mathrm{f}}}{k_{\mathrm{s}}}+1-\varepsilon\right)
$$

resulting in $e_{1}=0.753$ and $e_{2}=0.267 . e_{1}$ and $e_{2}$ depend strongly on the morphology. This can be seen when comparing $k_{\mathrm{e}}$ of the 20 ppi and the 10 ppi foams analyzed in Ref. [20], both having the same $\varepsilon$ and a rather sharp pore size peak (peak width $\approx 0.5 d_{\text {nom }}$ ). The 20 ppi foam shows a larger $A_{0}$. For $k_{\mathrm{f}} / k_{\mathrm{s}} \approx 10^{-1}$ they differ by nearly $6 \%$ and at $k_{\mathrm{f}} / k_{\mathrm{s}} \approx 10^{-4}$ they differ by up to $40 \%$. Obviously, the smaller $k_{\mathrm{f}} / k_{\mathrm{s}}$, the more important becomes the phase distribution. When using the RPC foam as solar absorber, larger $k_{\mathrm{e}}$ are preferred since they allow for faster heat transfer rate and a more uniform heating.

3.3 Interfacial Heat Transfer Coefficient. The heat flux from the solid to the fluid phase is given by

$$
q=h_{\mathrm{sf}} A_{\mathrm{sf}}\left(T_{\text {interface }}-T_{\mathrm{mf}}\right)
$$

The coupled continuity, momentum, and energy equations are solved within the fluid phase of a square duct containing a sample of the foam by using a computational fluid dynamics (CFD) code [34] to obtain the temperature distribution in the fluid phase and the heat fluxes through the solid-fluid interface.

The boundary conditions are

$$
\overline{\mathbf{u}}=0, \quad T=T_{\text {sf }} \quad \text { at the solid-fluid interface }
$$

$\overline{\mathbf{u}} \cdot \hat{\mathbf{n}}=0, \quad \hat{\mathbf{n}} \cdot \nabla \overline{\mathbf{u}}=0, \quad \mathbf{q}^{\prime \prime} \cdot \hat{\mathbf{n}}=0 \quad$ at the sample later walls

$$
\begin{gathered}
\overline{\mathbf{u}} \cdot \hat{\mathbf{n}}=-u_{0}, \quad T=T_{0} \quad \text { at the inlet } \\
p=p_{\text {atm }} \quad \text { at the outlet }
\end{gathered}
$$

$h_{\mathrm{sf}}$ is then determined as a function of Pr and Re by using

$$
h_{\mathrm{sf}}=\frac{\int_{A_{\mathrm{sf}}} q^{\prime \prime} \mathrm{d} A_{\mathrm{sf}}}{A_{\mathrm{sf}} \cdot \Delta T_{\mathrm{lm}}}
$$

A sample with dimensions of $11.4 \times 11.4 \times 3.78 \mathrm{~mm}^{3}$, corresponding to $380 \times 380 \times 126$ voxels, is investigated. Convergence was achieved for the termination residual RMS of the iterative solution below $10^{-4}$ and for the maximal mesh element length of $90 \mu \mathrm{m}$ (corresponding to $0.07 d_{\text {nom }}$ ), resulting in $5.6 \times 10^{7}$ tetrahedral elements. The mesh is generated with an in-house mesh generator for unstructured body-fitted grids. The mesh generator covers the domain by tetrahedral elements and subsequently refines the elements at the phase boundary. Finally, a rounding, cutting, and smoothing process is made to achieve an accurate domain representation [21]. Two quad-core Intel Xeon $2.5 \mathrm{GHz}$ processors and 32 Gbytes RAM are used to solve the equations in approximately $10 \mathrm{~h}$.

$\mathrm{Nu}$ is shown in Fig. 9 as a function of $\mathrm{Pr}$ and Re. Assuming a correlation of the form $\mathrm{Nu}=a_{0}+a_{1} \operatorname{Re}^{a_{2}} \operatorname{Pr}^{a_{3}}$, least-square fitting leads to $(\mathrm{RMS}=0.817)$

$$
\mathrm{Nu}=6.820+0.198 \operatorname{Re}^{0.788} \operatorname{Pr}^{0.606}
$$

These results compare well to those obtained experimentally for a 10 ppi foam [35].

3.4 Influence of $l_{\mathrm{REV}}$. Normalized porosity, extinction coefficient, and conductivity are plotted in Fig. 10 as a function of 


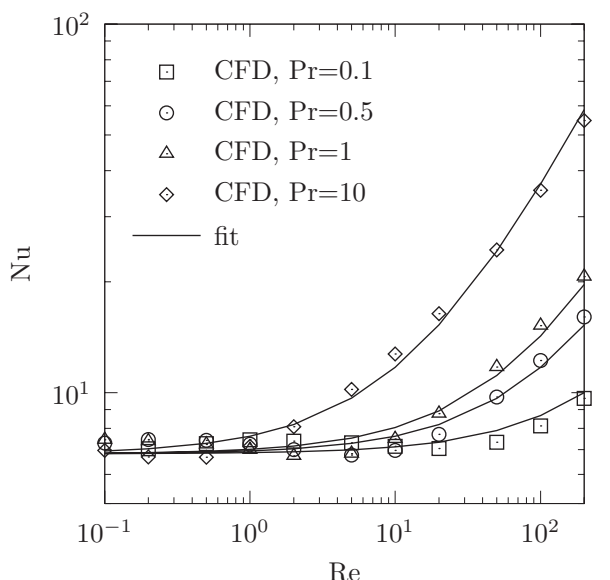

Fig. 9 Computed (points) and fitted (lines) Nu number as a function of Re and Pr numbers

$l / d_{\text {nom }}$ varying between 0 and 6 . All three parameters converge for approximately $l_{\mathrm{REV}}=3.5 d_{\text {nom }}$, with $\gamma= \pm 0.02, \pm 0.02$, and \pm 0.15 , respectively.

\section{Mass Transfer Characteristics}

4.1 Permeability and Dupuit-Forchheimer Coefficient. Darcy's law [36] and its extensions [37,38] are applied for a fluid flow in an averaged isotropic porous medium

$$
\nabla p=-\frac{\mu}{K} \overline{\mathbf{u}}_{\mathrm{D}}-F_{\mathrm{DF}} \rho \overline{\mathbf{u}}_{\mathrm{D}}\left|\overline{\mathbf{u}}_{\mathrm{D}}\right|
$$

Nondimensionalization of Eq. (25) for the 1D case yields

$$
\frac{\nabla p d^{2}}{\mu u_{\mathrm{D}}}=\Pi_{\mathrm{pg}}=-\frac{d^{2}}{K}-F_{\mathrm{DF}} d \operatorname{Re}=-c_{0}-c_{1} \operatorname{Re}
$$

The continuity and momentum equations are solved [34] within the fluid phase of a square duct containing a sample of the foam to obtain the velocity and pressure distributions for the following boundary conditions

$$
\overline{\mathbf{u}}=0 \quad \text { at the solid-fluid interface }
$$

$\overline{\mathbf{u}} \cdot \hat{\mathbf{n}}=0, \quad \hat{\mathbf{n}} \cdot \nabla \overline{\mathbf{u}}=0 \quad$ at the sample lateral walls

$$
\overline{\mathbf{u}} \cdot \hat{\mathbf{n}}=-u_{0} \quad \text { at the inlet }
$$

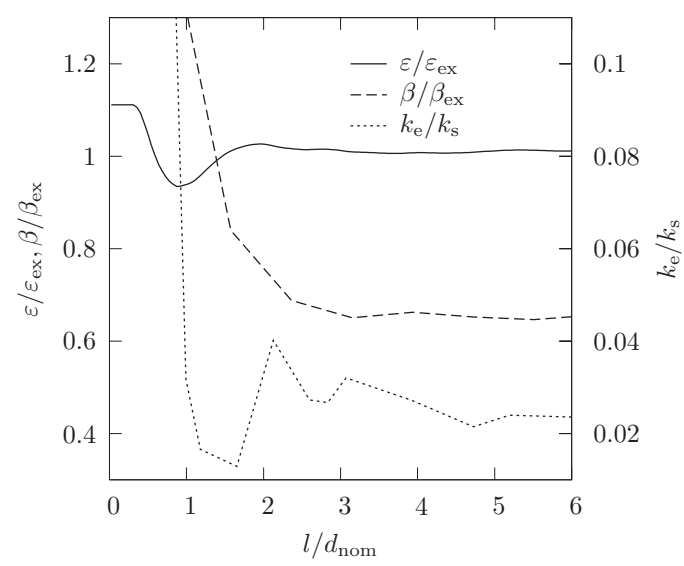

Fig. 10 Normalized porosity, extinction coefficient, and effective conductivity for cubic volumes with edge lengths I

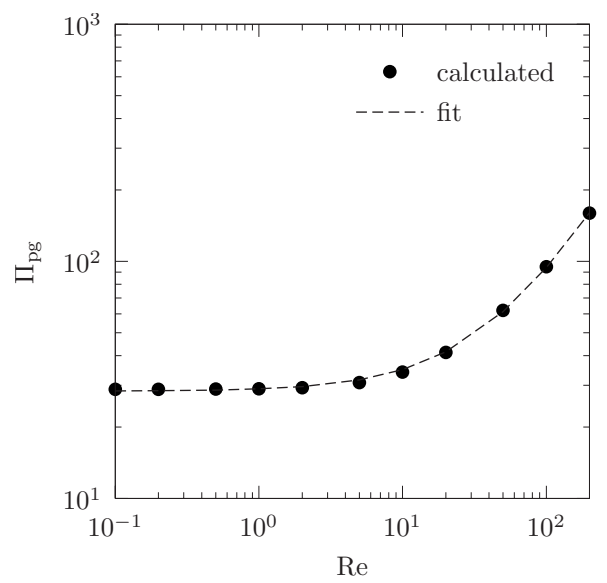

Fig. 11 Dimensionless pressure gradient as a function of Re number

$$
p=p_{\text {atm }} \text { at the outlet }
$$

A $11.4 \times 11.4 \times 3.78 \mathrm{~mm}^{3}$ sample, corresponding to $380 \times 380$ $\times 252$ voxels, is investigated. Convergence is achieved for the termination residual RMS of the iterative solution below $10^{-4}$ and for the maximal mesh element length of $180 \mu \mathrm{m}$ (corresponding to $\left.0.14 d_{\text {nom }}\right)$, resulting in $5.6 \times 10^{7}$ tetrahedral elements. The dimensionless pressure gradient is plotted as a function of Re in Fig. 11. Least-square fitting (RMS $=0.566)$ results in $c_{0}=28.334$ and $c_{1}=0.659$, which correspond to $K=5.69 \times 10^{-8} \mathrm{~m}^{2}$ and $F_{\mathrm{DF}}$ $=519.0 \mathrm{~m}^{-1}$. Permeability values are compared with those obtained for the Hagen-Poisseuille model $\left(4.59 \times 10^{-8} \mathrm{~m}^{2}\right)$ [14], the Carman-Konzeny model $\left(8.69 \times 10^{-8} \mathrm{~m}^{2}\right)[14,15]$, the fibrous bed models $\left(8.97 \times 10^{-7} \mathrm{~m}^{2}\right)$ [15], and a model proposed by Macdonald et al. $\left(5.34 \times 10^{-8} \mathrm{~m}^{2}\right)$ [39]. The latter model yields $F_{\mathrm{DF}}$ $=544.2 \mathrm{~m}^{-1}$. The nonlinear term in Eq. (25) comes into play for $\operatorname{Re}>1$. Increased pore size leads to reduced pressure loss and consequently to a larger $K$ and a smaller $F_{\mathrm{DF}}$, as observed by comparing the SiSiC RPC to the Rh-coated $\mathrm{SiC}$ from Ref. [21].

4.2 Tortuosity and Residence Time Distributions. Tortuosity is defined as the ratio of the real length of the connected pore channels to the thickness of the porous sample in the main flow direction

$$
\tau=\frac{l_{\text {path }}}{l_{\text {sample }}}
$$

The residence time required for a particle to flow through the porous sample is defined as

$$
t=\int_{l_{\text {path }}} \frac{1}{u} \mathrm{~d} l
$$

The velocity distributions obtained in the Sec. 4.1 are now used to determine the tortuosity and residence time distributions. They are shown in Fig. 12 for $\mathrm{Re}=0.1,1,10$, and 100. Mean tortuosity is 1.07. The peak of the tortuosity distribution shifts toward $\tau=1$ with increasing Re. Mean residence time is plotted in Fig. 13. All stream lines generated are able to pass through the sample, as no recirculation zones, dead ends, or flow reversals are observed. In most chemical applications, large $\tau$, smaller Re, and consequently large $t$ are preferred to allow for complete reaction conversion.

4.3 Dispersion Tensor. The governing equation coupling the convection and diffusion in the fluid phase is [40] 

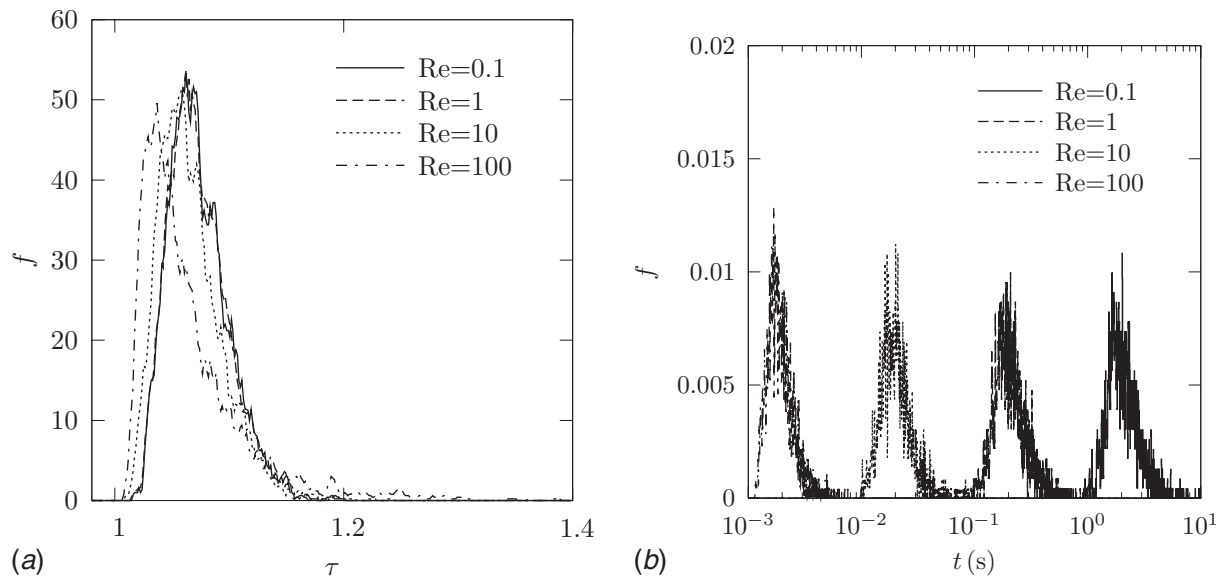

Fig. 12 (a) Tortuosity and (b) residence time distributions for four selected Re numbers of fluid flow through the RPC foam

$$
\frac{\partial c}{\partial t}+\nabla(c \overline{\mathbf{u}})-\nabla(\overline{\overline{\mathbf{D}}} \cdot \nabla c)=0
$$

The dispersion tensor $\mathbf{D}$ in an isotropic medium can be decomposed in parallel and transverse components, $D_{\mathrm{II}}$ and $D_{\perp}$, respectively. For zero molecular diffusion

$$
D_{i}=b_{0} \operatorname{Re}^{b_{1}}
$$

with $b_{0}$ and $b_{1}$ constants. The solution of Eq. (33) links $\mathbf{D}$ to the standard deviation of the normally distributed concentration $c$ [41]

$$
S_{c}=\sqrt{2 D_{i} t}
$$

$D_{x}, D_{y}$, and $D_{z}$ are determined by following 2500 streamlines through the foam, registering their spatial displacement at a specific time instant (e.g., in $z$-direction: $\Delta z=z(t)-z\left(t_{0}\right)$ ), and subsequently fitting the registered distribution to a standard Gauss distribution. The normalized dispersion tensor components are shown in Fig. 14 as a function of Re. $D_{x}$ and $D_{y}$ are equal to the transverse component $D_{\perp} ; D_{z}$ is equal to the parallel component $D_{\mathrm{II}}$. Fitting to Eq. (34) yields

$$
\begin{aligned}
& \frac{D_{\perp}}{\nu}=\left\{\begin{array}{ll}
6.560 \times 10^{-3} \operatorname{Re} & \operatorname{Re} \leq 5 \\
4.896 \times 10^{-3} \operatorname{Re}^{1.104} & \operatorname{Re}>5
\end{array} \quad\left(\mathrm{RMS}=6.0 \times 10^{-6}\right)\right. \\
& \frac{D_{\mathrm{II}}}{\nu}=\left\{\begin{array}{ll}
6.297 \times 10^{-1} \operatorname{Re} & \operatorname{Re} \leq 5 \\
7.045 \times 10^{-1} \mathrm{Re}^{0.942} & \operatorname{Re}>5
\end{array} \quad\left(\mathrm{RMS}=4.2 \times 10^{-5}\right)\right.
\end{aligned}
$$

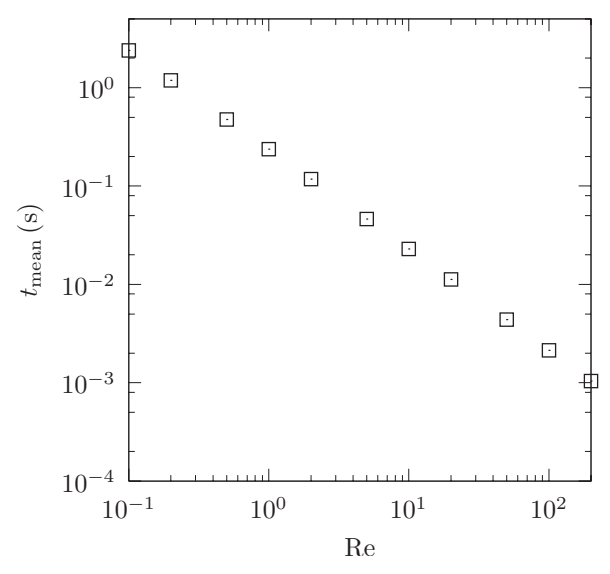

Fig. 13 Mean residence time as a function of Re number

\section{Summary and Conclusions}

We have numerically computed the effective heat and mass transport properties of a nonhollow RPC foam made of SiSiC, whose exact 3D geometry was determined by computer tomography. Computed porosity was 0.91 and compared well to experimentally measured value of $0.90 \pm 0.02$. Computed specific surface was $1367 \mathrm{~m}^{-1}$ and increased by $20 \%$ when increasing the scan resolution by a factor of 2 as smaller surface irregularities were resolved. Computed pore size distribution showed a sharp peak of approximately $0.5 d_{\text {nom }}$ and a mean diameter of $1.3 d_{\text {nom. }}$. REV determined by porosity, extinction coefficient, and conductivity calculations on subsequently growing volumes was $87.8 \mathrm{~mm}^{3}$. Radiative properties were determined from the extinction length and cosine of incidence distributions by applying the collision-based MC method. The computed extinction coefficient of $431 \mathrm{~m}^{-1}$ agreed quantitatively to the experimentally measured one estimated by measuring the transmitted radiative flux with a spectroscopy system. Computed scattering functions showed a large backward scattering peak for diffusely-reflecting surfaces and isotropic scattering behavior for specularly-reflecting surfaces. The scattering coefficient was a function of the surface reflectivity and determined to be $63 \mathrm{~m}^{-1}$. The effective conductivity was calculated by solving the heat conduction equation within both phases by $\mathrm{FV}$ and fitted to a combination of parallel and serial slab models. For $k_{\mathrm{f}} / k_{\mathrm{s}}<10^{-4}, k_{\mathrm{e}}$ remained constant and approximately $0.022 k_{\mathrm{s}}$. The heat transfer coefficient was calculated by solving the continuity, momentum, and energy governing

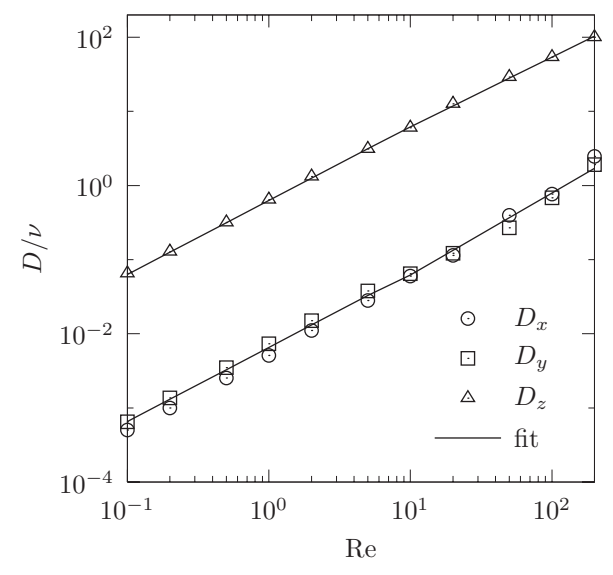

Fig. 14 Normalized dispersion tensor as a function of Re for the RPC foam 
equations within the fluid phase by FV. A Re and Pr dependent $\mathrm{Nu}$ correlation of the form $\mathrm{Nu}=6.820+0.198 \mathrm{Re}^{0.788} \mathrm{Pr}^{0.606}$ was fitted $(\mathrm{RMS}=0.817)$. This correlation strongly depended on the morphology. Computed permeability and Dupuit-Forchheimer coefficient, determined based on the pressure and velocity distribution within the fluid, were $K=5.67 \times 10^{-8} \mathrm{~m}^{2}$ and $F_{\mathrm{DF}}=519.0 \mathrm{~m}^{-1}$ and compared well to the values found by applying different models available in the literature. Tortuosity distribution calculations resulted in a mean tortuosity of 1.07. Obviously, the mean residence time decreased with increasing Re. Neglecting molecular dispersion, a Re dependent function of the dispersion tensor was obtained by comparing the calculated spatial displacement distribution of streamlines within the foam to a Gaussian distribution.

The CT-based methodology is able to accurately account for the morphology of complex porous media, and, when coupled to Monte Carlo and CFD numerical techniques, provides pore-level solutions of the energy and fluid flow governing equations. The effective transport properties can be used in a continuum-scale heat and mass transfer model, which, in turn, may be used for the design and optimization of components for high-temperature applications. A follow-up study will determine the limits of applicability of combined conduction-convection-radiation computations utilizing the effective thermal properties.

\section{Acknowledgment}

This work has been financially supported by the Swiss National Science Foundation under Contract No. 200021-115888 and by the European Commission under Contract No. 212470 (Project HYCYCLES). We thank C. Suter for the technical support with the spectroscopy measurements and F. Marone for the technical support at SLS.

\section{Nomenclature}

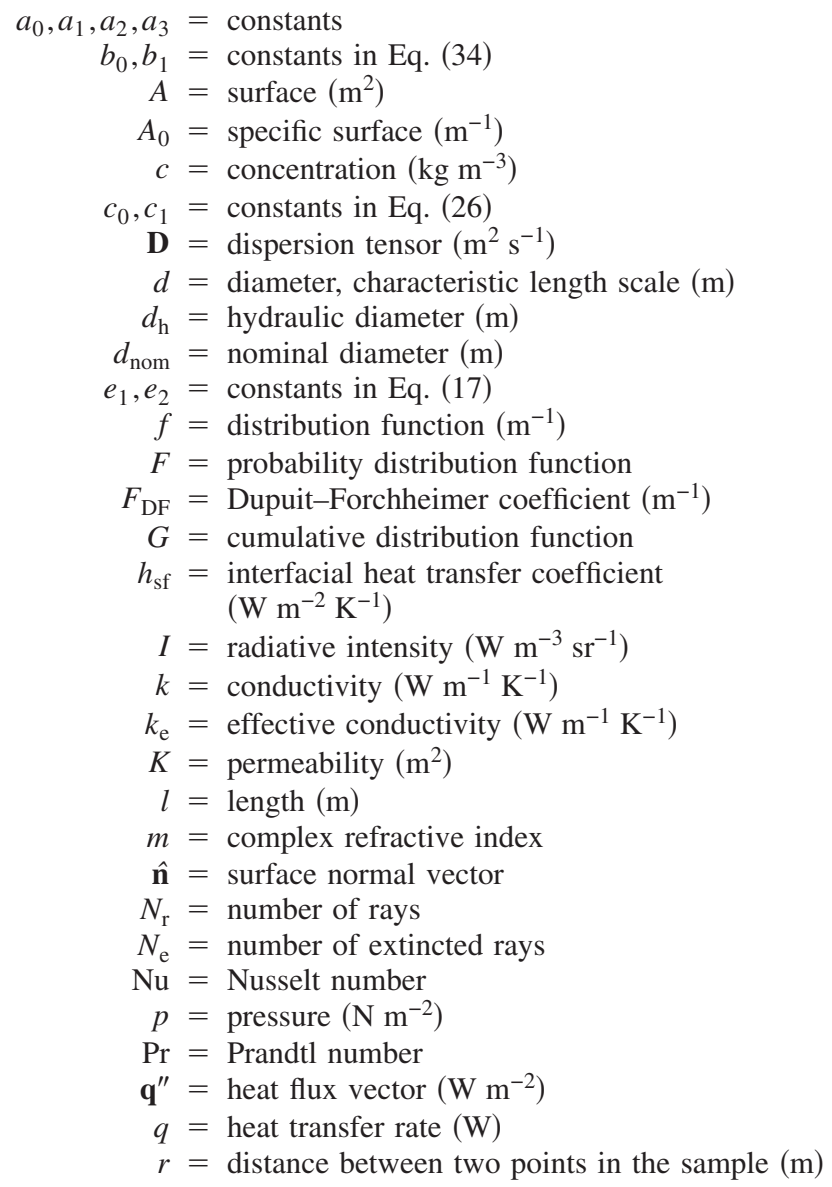

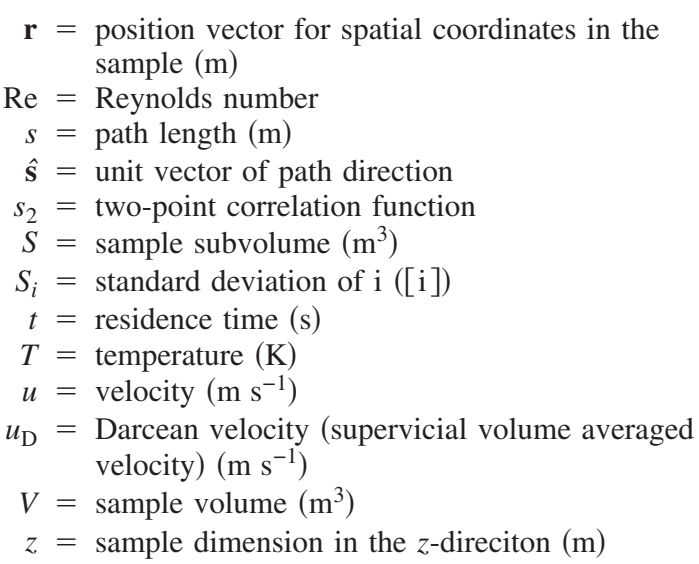

Greek

$$
\begin{aligned}
\alpha & =\text { absorption values of tomographic scans }\left(\mathrm{m}^{-1}\right) \\
\beta & =\text { extinction coefficient }\left(\mathrm{m}^{-1}\right) \\
\delta & =\text { Dirac delta function } \\
\gamma & =\text { half bandwidth for REV determination } \\
\varepsilon & =\text { porosity } \\
\kappa & =\text { absorption coefficient }\left(\mathrm{m}^{-1}\right) \\
\lambda & =\text { radiation wavelength }\left(\mathrm{m}^{-1}\right. \\
\mu & =\text { dynamic viscosity }\left(\mathrm{kg} \mathrm{m}^{-1} \mathrm{~s}^{-1}\right) \\
\mu_{\mathrm{in}} & =\text { cosine of incidence angle } \\
\mu_{\mathrm{r}} & =\text { cosine of reflection angle } \\
\mu_{\mathrm{s}} & =\text { cosine of scattering angle } \\
\nu & =\text { kinematic viscosity }\left(\mathrm{m}^{2} \mathrm{~s}^{-1}\right) \\
\varphi_{\mathrm{d}} & =\text { difference azimutal angle, } \varphi_{\mathrm{d}}=\varphi_{\text {in }}-\varphi_{\mathrm{r}}(\mathrm{rad}) \\
\rho^{\prime \prime} & =\text { bidirectional reflectivity }\left(\mathrm{sr}^{-1}\right) \\
\Pi_{\mathrm{pg}} & =\text { dimensionless pressure gradient } \\
\sigma_{\mathrm{s}} & =\text { scattering coefficient }\left(\mathrm{m}^{-1}\right) \\
\tau & =\text { tortuosity } \\
\Phi & =\text { scattering phase function } \\
\psi & =\text { pore-scale indicator function }(1=\text { void phase; } 0 \\
\Omega & =\text { = solid phase) }
\end{aligned}
$$

Subscripts

$$
\begin{aligned}
\mathrm{am} & =\text { arithmetic mean } \\
\mathrm{atm} & =\text { atmospheric } \\
\mathrm{b} & =\text { blackbody } \\
\mathrm{ex} & =\text { experimental } \\
\mathrm{f} & =\text { fluid } \\
\mathrm{in} & =\text { incident } \\
\mathrm{lm} & =\text { logarithmic mean } \\
\mathrm{mf} & =\text { mean fluid } \\
\mathrm{op} & =\text { opening } \\
\mathrm{s} & =\text { solid } \\
\mathrm{sf} & =\text { solid-fluid boundary } \\
\mathrm{t} & =\text { threshold } \\
\mathrm{tot} & =\text { total } \\
0 & =\text { initial }
\end{aligned}
$$

\section{References}

[1] Nettleship, I., 1996, “Applications of Porous Ceramics," Adv. Ceram. Mater., 122, pp. 305-324.

[2] Matthews, A. L., 1996, "Ceramic Filters for the Cast Metal Industry," Adv Ceram. Mater., 122, pp. 293-304.

[3] van Setten, B., Bremmer, J., Jelles, S., Makkee, M., and Moulijn, J., 1999 "Ceramic Foam as a Potential Molten Salt Oxidation Catalyst Support in the Removal of Soot From Diesel Exhaust Gas," Catal. Today, 53, pp. 613-621.

[4] Barra, A., Diepvens, G., Ellzey, J., and Henneke, M., 2003, "Numerical Study of the Effects of Material Properties on Flame Stabilization in a Porous Burner," Combust. Flame, 134, pp. 369-379.

[5] Dhamrat, R., and Ellzey, J., 2006, "Numerical and Experimental Study of the Conversion of Methan to Hydrogen in a Porous Media Reactor," Combust. Flame, 144, pp. 698-709.

[6] Fend, T., Hoffschmidt, B., Pitz-Paal, R., Reutter, O., and Rietbrock, P., 2004 
"Porous Materials as Open Volumetric Solar Receivers: Experimental Determination of Thermophysical and Heat Transfer Properties," Energy, 29, pp. 823-833.

[7] Buck, R., Muir, J. F., Hogan, R. E., and Skocypec, R. D., 1991, "Carbon Dioxide Reforming of Methane in a Solar Volumetric Receiver/Reactor: The CAESAR Project," Solar Energy Materials, 24, pp. 449-463.

[8] Chavez, J. H., and Chaza, C., 1991, "Testing of Porous Ceramic Absorber for a Volumetric Air Receiver," Solar Energy Materials, 24, pp. 172-181.

[9] Loretz, M., Coquard, R., Baillis, D., and Maire, D., 2008, "Metallic Foams: Radiative Properties/Comparison Between Different Models," J. Quant. Spectrosc. Radiat. Transf., 109, pp. 16-27.

[10] Fu, X., Viskanta, R., and Gore, J. P., 1997, "A Model for the Volumetric Radiation Characteristics of Cellular Ceramics," Int. Commun. Heat Mass Transfer, 24, pp. 1069-1082.

[11] Boomsma, K., and Poulikakos, D., 2001, "On the Effective Thermal Conductivity of Three-Dimensionally Structured Fluid-Saturated Metal Foam,” Int. J. Heat Mass Transfer, 44, pp. 827-836.

[12] Bhattacharya, A., Calmidi, V., and Mahajan, R., 2002, "Thermophysical Properties of High Porosity Metal Foams,” Int. J. Heat Mass Transfer, 45, pp. 1017-1031.

[13] Fu, X., Viskanta, R., and Gore, J. P., 1998, "Prediction of Effective Thermal Conductivity of Cellular Ceramics," Int. Commun. Heat Mass Transfer, 25, pp. 151-160.

[14] Kaviany, M., 1995, Principles of Heat Transfer in Porous Media, SpringerVerlag, New York.

[15] Dullien, F., 1979, Porous Media Fluid Transport and Pore Structure, Academic, New York.

[16] Chen, C., 1955, "Filtration of Aerosols by Fibrous Media," Chem. Rev. (Washington, D.C.), 55, pp. 595-623.

[17] Rintoul, M., Torquato, S., Yeong, C., Keane, D., Erramilli, S., Jun, Y., Dabbs, D., and Aksay, I., 1996, "Structure and Transport Properties of a Porous Magnetic Gel Via X-Ray Microtomography,” Phys. Rev. E, 54, pp. 2663-2669.

[18] Petrasch, J., Wyss, P., Stämpfli, R., and Steinfeld, A., 2008, "TomographyBased Multiscale Analyses of the 3D Geometrical Morphology of Reticulated Porous Ceramics," J. Am. Ceram. Soc., 91, pp. 2659-2665.

[19] Petrasch, J., Wyss, P., and Steinfeld, A., 2007, "Tomography-Based Monte Carlo Determination of Radiative Properties of Reticulate Porous Ceramics," J. Quant. Spectrosc. Radiat. Transf., 105, pp. 180-197.

[20] Petrasch, J., Schrader, B., Wyss, P., and Steinfeld, A., 2008, "TomographyBased Determination of the Effective Thermal Conductivity of Reticulate Porous Ceramics," ASME J. Heat Transfer, 130, p. 032602.

[21] Petrasch, J., Meier, F., Friess, H., and Steinfeld, A., 2008, "Tomography Based Determination of Permeability, Dupuit-Forchheimer Coefficient, and Interfacial Heat Transfer Coefficient in Reticulate Porous Ceramics," Int. J. Heat Fluid Flow, 29, pp. 315-326.

[22] Haussener, S., Lipiński, W., Petrasch, J., Wyss, P., and Steinfeld, A., 2009 "Tomographic Characterization of a Semitransparent-Particle Packed Bed and Determination of Its Thermal Radiative Properties," ASME J. Heat Transfer,
131, p. 072701

[23] Noglik, A., Roeb, M., Rzepczyk, T., Hinkley, J., Sattler, C., and Pitz-Paal, R. 2009, "Solar Thermochemical Generation of Hyrogen: Develpment of a Receiver Reactor of the Decomposition of Sulfuric Acid," J. Sol. Energy Eng., 131, p. 011003

[24] Gonzalez, R. C., and Woods, R. E., 2008, Digital Image Processing, 3rd ed., Prentice-Hall, Upper Saddle River, NJ.

[25] Weszka, Z., 1978, "A Survey of Threshold Selection Techniques," Comput. Graph. Image Process., 7, pp. 259-265.

[26] Streun, A., Böge, A., Dehler, M., Gough, C., Joho, W., Korhonen, T., Lüdeke, A., Marchand, P., Muñoz, M., Pedrozzi, M., Rivkin, L., Schilcher, T., Schlott, V., Schulz, L., and Wrulich, A., 2001, "Commissioning of the Swiss Light Source," Proceedings of the 2001 Particle Accelerator Conference, P. Lucas and S. Webber, eds., IEEE, Piscataway, NJ, pp. 224-226.

[27] Berryman, J., and Blair, S., 1986, "Use of Digital Image Analysis to Estimate Fluid Permeability of Porous Material: Application of Two-Point Correlation Functions," J. Appl. Phys., 60, pp. 1930-1938.

[28] Palik, E. D., 1998, Handbook of Optical Constants of Solids II, Elsevier, New York.

[29] Lipiński, W., Petrasch, J., and Haussener, S., 2009, "Application of the Spatial Averaging Theorem to Radiative Heat Transfer in Two-Phase Media," J. Quant. Spectrosc. Radiat. Transf., 111, pp. 253-258.

[30] Tancrez, M., and Taine, J., 2004, "Direct Identification of Absorption and Scattering Coefficients and Phase Function of a Porous Medium by a Monte Carlo Technique," Int. J. Heat Mass Transfer, 47, pp. 373-383.

[31] Modest, M., 2003, Radiative Heat Transfer, 2nd ed., Academic, San Diego, CA.

[32] Touloukian, Y., 1970, Thermophysical Properties of Matter, Plenum, New York.

[33] Coray, P., Lipiński, W., and Steinfeld, A., 2008, "Experimental and Numerical Determination of Thermal Radiative Properties of ZnO Particulate Media," ASME J. Heat Transfer, in press.

[34] Ansys Inc., 2006, ANSYs-CFX, www.ansys.com

[35] Buck, R., 2000, "Massenstrom-Instabilitäten bei volumetrischen ReceiverReaktoren," Ph.D. thesis, Universität Stuttgart, Germany.

[36] Darcy, H., 1856, Les Fontaines Publiques de la ville de Dijon, Dalmont, Paris

[37] Dupuit, J., 1863, Etudes theoretiques et practiques sur le mouvement des eaux, Dunond, Paris.

[38] Forchheimer, P., 1901, "Wasserbewegung durch den Boden," Z. Ver. Dtsch. Ing., 45, pp. 1782-1788.

[39] Macdonald, I. F., El-Sayed, M. S., Mow, K., and Dullien, F. A. L., 1979 , "Flow Through Porous Media-The Ergun Equation Revisited," Ind. Eng. Chem. Fundam., 18, pp. 199-208.

[40] Hirsch, C., 2007, Numerical Computation of Internal and External Flows, 2nd ed., Elsevier, Amsterdam.

[41] Salles, J., Thovert, J.-F., Delannay, R., Prevors, L., Auriault, J.-L., and Adler, P. M., 1993, "Taylor Dispersion in Porous Media. Determination of the Dispersion Tensor," Phys. Fluids, 5, pp. 2348-2376. 